ARTICLE

https://doi.org/10.1038/s41467-019-09868-6

\title{
Programmable terahertz chip-scale sensing interface with direct digital reconfiguration at sub-wavelength scales
}

\author{
Xue Wu${ }^{1}$, Huaixi Lu $\mathbb{1}^{1}$ \& Kaushik Sengupta ${ }^{1}$
}

The ability to sense terahertz waves in a chip-scale technology operable at room temperature has potential for transformative applications in chemical sensing, biomedical imaging, spectroscopy and security. However, terahertz sensors are typically limited in their responsivity to a narrow slice of the incident field properties including frequency, angle of incidence and polarization. Sensor fusions across these field properties can revolutionize $\mathrm{THz}$ sensing allowing robustness, versatility and real-time imaging. Here, we present an approach that incorporates frequency, pattern and polarization programmability into a miniaturized chipscale $\mathrm{THz}$ sensor. Through direct programming of a continuous electromagnetic interface at deep subwavelength scales, we demonstrate the ability to program the sensor across the spectrum (0.1-1.0 THz), angle of incidence and polarization simultaneously in a single chip implemented in an industry standard 65-nm CMOS process. The methodology is compatible with other technology substrates that can allow extension of such programmability into other spectral regions.

\footnotetext{
${ }^{1}$ Department of Electrical Engineering, Princeton University, Princeton, NJ 08544, USA. Correspondence and requests for materials should be addressed to K.S. (email: kaushiks@princeton.edu)
} 
$\mathrm{T}$ he terahertz spectrum, occupying the frequency range between 0.3 and $3 \mathrm{THz}$, has potential for transformative applications in communication, sensing, spectroscopy, and imaging due to its desirable properties such as non-ionizing photon energy, penetration capability through optically opaque materials, unique spectral signatures for large bio-molecules and chemicals $^{1-9}$. To enable this diverse set of applications, there has been a concerted effort in the research community to miniaturize complex $\mathrm{THz}$ systems into chip-scale form that are operable at room temperature. Wedged between the microwave band and the infrared spectrum, this effort has spanned across a large array of substrates ranging from solid-state to photonic devices ${ }^{10-14}, 2 \mathrm{D}$ nano-materials ${ }^{15,16}$, quantum-cascade lasers $(\mathrm{QCLs})^{17-19}$, microbolometers ${ }^{20,21}$, nanowires ${ }^{22}$, metamaterials ${ }^{23}$, and ultrafast photoconductive materials ${ }^{24,25}$. In recent years, even silicon, particularly complementary-metal-oxide-semiconductor (CMOS) based integrated circuits and chips have been demonstrated in the frequency range with power generation capability in the range of 100 s of $\mu \mathrm{W}^{26-29}$ and detection capability with sensitivities (noiseequivalent-power) in the sub-100 $\mathrm{pW} / \sqrt{\mathrm{Hz}}$ region $^{30-37}$. The field-effect-based devices can detect $\mathrm{THz}$ waves at frequencies beyond their cutoff frequencies by exploiting nonlinearities in the operation regime through a non quasistatic plasma-wave excitation by the incident $\mathrm{THz}$ waves ${ }^{38}$. They also exhibit orders of magnitude much faster response times and higher pixel integration capability compared with microbolometers ${ }^{32}$. This has been a significant advancement, as it not only makes possible $\mathrm{THz}$ systems (below $1 \mathrm{THz}$ ) compact and battery operable at room temperature, but also exploits the economics of scale of semiconductor fabrication to enable complex $\mathrm{THz}$ systems in a costeffective fashion.

A limiting factor in all of these classes of $\mathrm{THz}$ sensors is that they are typically sensitive to a narrow set of the incident field properties, i.e., including a limited spectral range, angle of incidence, and polarization. This has hindered deployment of $\mathrm{THz}$ sensors in practical applications where single modality sensing across any field property is not robust enough. Due to the spectrally sensitive nature of scattering and penetration through optically opaque objects in these frequency ranges, sensor fusions exploiting frequency, pattern and polarization diversity, will become increasingly important for high-performance sensing applications. Prior works have demonstrated merging imaging with spectroscopic sensing, exploiting the unique advantages in the $\mathrm{THz}$ regime, to clearly differentiate the advantages against other spectral regions ${ }^{39-41}$. We are seeing a similar evolution in the neighboring frequencies, where sensor fusions particularly combining millimeter-wave, infra-red and optical frequencies is becoming critical to enable effective and robust understanding of the environment for autonomous vehicles and systems. In a similar fashion, the ability to extract information across the incident $\mathrm{THz}$ field properties can allow a much richer sensing interface ${ }^{42,43}$.

Enabling such programmability in the $\mathrm{THz}$ spectrum, particulary in chip-scale form, is very challenging. Limited reconfigurability in $\mathrm{THz}$ systems have been demonstrated in mechanically and optically controlled metamaterials ${ }^{43,44}$, microelectromechanically actuated slits for $\mathrm{THz}$ modulation ${ }^{45}$, thermally tunable $\mathrm{THz}$ filters ${ }^{46}$ and phase-change material ${ }^{47}$, graphene-based switchable high-impedance surface (HIS) ${ }^{48,49}$, and graphene-based multi-input-multi-output antenna array ${ }^{50}$. The key to realize such a rich sensing interface is to allow simultaneous programmability across all the three incident field properties and across such a wide variation range. In addition, integration in a substrate compatible with semiconductor fabrication processes is also important to allow for low-cost and wide- scale deployments of such sensors. This is particularly challenging, since the $\mathrm{THz}$ frequencies can far exceed the cutoff frequencies of such devices making any form of reconfigurability very limited and inefficient.

In this paper, we present our approach toward a universal $\mathrm{THz}$ sensing surface which can reconfigure its responsivity to spectrum $(0.1-1.0 \mathrm{THz})$, incidence direction and polarization. We demonstrate the approach in a single chip implemented in an industry standard $65-\mathrm{nm}$ CMOS process with $f_{\max } \approx 0.25 \mathrm{THz}$. The metric $f_{\max }$ designates the frequency where the unilateral gain of the device falls to one ${ }^{1}$. Therefore, this distributed approach, realized with devices operating up to four times their cutoff frequencies, can be translated to other semiconductor platforms for programmability in other spectral ranges. While preliminary results on the sensor response at $\mathrm{THz}$ were presented in ref. ${ }^{51}$, this paper focuses on the design methodology of mapping subwavelength reconfiguration states to the set of incident field parameters including spectrum, angle of incidence and polarization. We also present measurement results in the implemented chip to demonstrate the tri-modal reconfigurability across $0.1-1.0 \mathrm{THz}$.

\section{Results}

Direct digital programming of $\mathrm{THz}$ surface. The ability to reconfigure against incident field properties opens up new dimensions to information that can be extracted from the sensor interface. Hyperspectral operation can enable simultaneously higher resolution acquisition with higher penetration depth for $3 \mathrm{D}$ imaging ${ }^{39}$ due to the spectrally dependent resolution and penetration depth of electromagnetic waves. The ability to electronically scan the beam pattern to various angles of incidence (pattern reconfigurability) can reduce image acquisition time by orders of magnitude circumventing slow mechanical raster scanning. In addition, such pattern diversity can also introduce new orthogonal measurements allowing computational-based techniques for real-time imaging ${ }^{51-53}$. A sensor that can program to orient its reception beam to different angles of incidence is tantamount to phased array operation. While phased arrays have been demonstrated at radio ${ }^{54}$ and optical frequencies ${ }^{55}$, terahertz operation of such arrays have been severely limited due to the unavailability of efficient active components such as amplifiers, phase shifters, and coherent sources which are the critical components of such a system ${ }^{1}$. Further, phased array operation is typically limited to a narrow range of frequencies where the discrete antenna spacings is $\sim \lambda / 2$ to avoid aliasing and grating lobes in unwanted directions. At the lower frequency end, spacings with $<\lambda / 2$ spacing reduces aperture area and typically suffers from inter-element mutual coupling that typically limits the array performance. Therefore, achieving simultaneous spectral and pattern reconfigurability has been very challenging, much more so at $\mathrm{THz}$ frequencies. In addition, the ability to reconfigure its polarization sensitivity is also important, since polarization rotation typically happens during transmission or reflectionbased imaging and in spectroscopy.

Fundamentally, the limitations of a $\mathrm{THz}$ sensor to these three $\mathrm{THz}$ field properties are dependent on the electromagnetic resonant nature of the interface, its scattering properties and the coupling to the detector, in whichever substrate the latter is realized. First, sensitivity to spectrum arises due to the resonant electromagnetic modes sustained on the antenna surface and the resonant nature of the antenna-detector interface ${ }^{37}$. The latter is designed to ensure optimal power transfer and impedance matching, that is typically guaranteed within a narrow range of frequencies. Broadband detection without frequency selectivity 
a
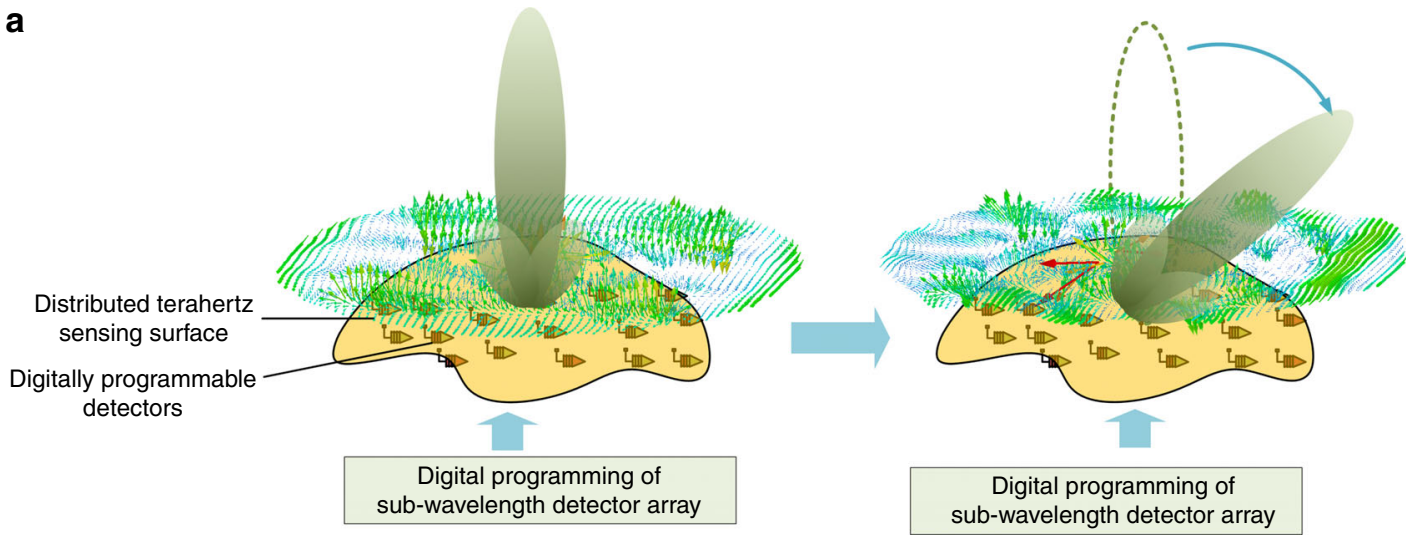

b

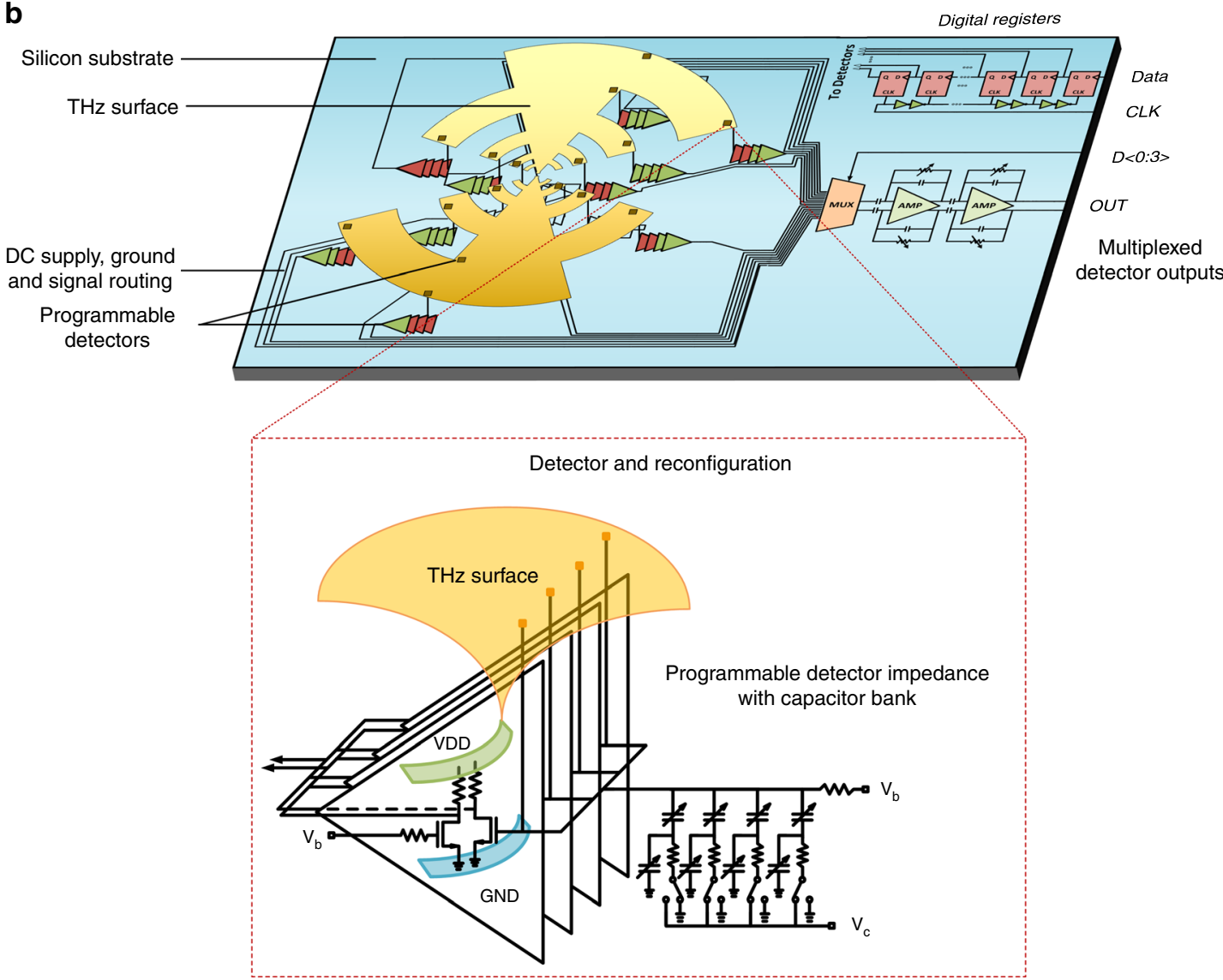

Fig. 1 Programmable terahertz chip-scale interface. a Concept of an universal terahertz sensing surface that maps a large discerte digital reconfiguration space to incident field properties including spectrum, angle of incidence and polarization. This is acheived by directly programming the surface currents induced on the interface at subwavelength scales. The incident power is absorbed in a distributed fashion at the sites of reconfiguration. $\mathbf{b}$ The system overview of the implemented terahertz sensor with a log-periodic tooth interface with 16 detectors distributed over the surface. Each site is reconfigurable with a coded capacitor bank that locally changes the boundary condition to redistribute the surface current distribution. This collectively programs the sensor response to allow for optimal reception against incident spectrum, polarization and angle of incidence

can be achieved in optical domain with photoconductive substrates $^{7}$, but the reception patterns are static and image acquisition requires bulky and complex optical assemblies, including femtosecond lasers. In addition to frequency selectivity, sensitivity to the other field properties namely angle of incidence and polarization also arises out of the antenna structure and the boundary conditions. Since a single-port antenna-detector system is reciprocal, its reception properties can be understood from its transmission properties. When excited, the antenna surface sustains a $2 \mathrm{D} \mathrm{THz}$ current distribution, that determines all its electromagnetic properties, including its frequency response, beam pattern, and polarization.

Traditional methods of reconfigurability that focus on the system by partitioning into its functional elements such as the antenna, the coupling network and the detectors are limited in their ability to efficiently achieve the desired parameters particularly at $\mathrm{THz}$ frequencies. Typically, such architectures focus on one aspect of reconfigurability against the incident field. While prototypical method of partitioning the design space and applying intuition-based approaches allow us to create 
a

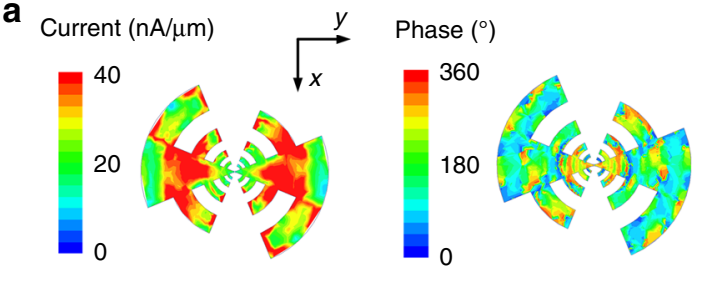

Broadside $x$-pol @ 0.3 THz

b

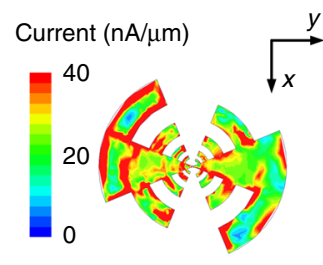

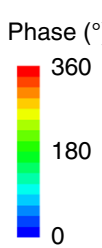

c

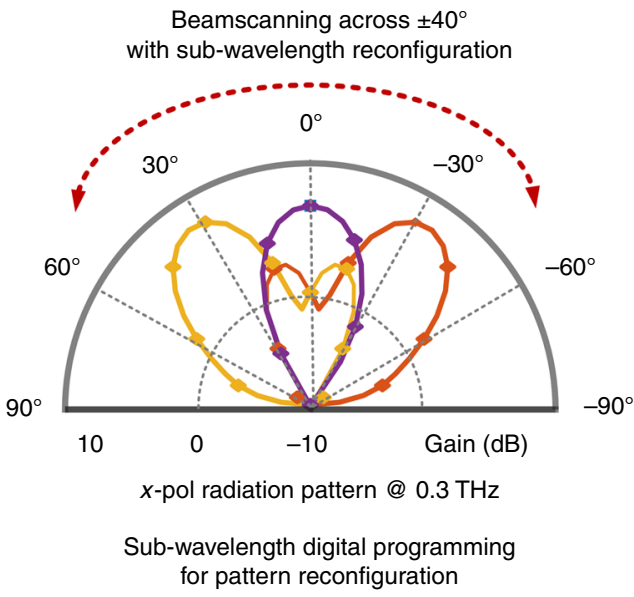

$45^{\circ} x$-pol @ $0.3 \mathrm{THz}$

Fig. 2 Direct digital programming of the interface against angle of incidence. The figure shows the distribution of amplitude and phase of the impressed surface current under incidence a $0.3 \mathrm{THz}$ at (a) broadside and (b) at $45^{\circ}$ under the optimal detector settings. c The simulated beam pattern of the collective absorption across the senor array shows the ability to orient it across various angles of incidence. Functionally, this is similar to a beam-scanning operation of phased arrays. Here, it is realized with a continuous aperture and subwavelength reconfiguration allowing pattern shaping and frequency agility simultaneously

a step-by-step design methodology, it also limits the space of possible architectures due to the complex interactions of several inter-dependent variables and properties. This is particularly true at $\mathrm{THz}$ frequencies where the individual device performance and variability itself is limited.

Since the electromagnetic properties of the $\mathrm{THz}$ interface is dependent on the THz surface current distribution it supports, the key concept in this work is to directly program the $2 \mathrm{D}$ distribution under $\mathrm{THz}$ field incidence. This is achieved with active devices placed at subwavelength scales that can simultaneously program and absorb the incident fields at the sites of reconfiguration. Figure 1 shows the concept where we move from a single-antenna-detector interface to a continuous aperture and distributed reconfiguration and absorption. The boundary conditions at each detector site is reconfigurable independently. This changes the local fields, reprograms the impressed current over the surface and redistributes the power distribution across the detector array. Through the complex interactions of the multiport distributed detector array, the $2 \mathrm{D}$ amplitude and phase distribution on the surface is changed. By independently programming the distributed detector array, a large set of $\mathrm{THz}$ sensor reception properties can be engineered. The goal of the reconfigurable $\mathrm{THz}$ sensor design is to map a subset of these large configuration states against optimal reception across frequency, pattern, and polarization.

Figure $1 \mathrm{~b}$ illustrates the architecture of the implemented reconfigurable $\mathrm{THz}$ surface. Each detector is realized with fieldeffect-transistors that rectifies the local $\mathrm{THz}$ field to produce a signal proportional to the local flux. Each detection site is programmable with a switched capacitor bank and a 4-bit thermometer code. With 16 detectors distributed over the surface, this results in $5^{16}$ possible reconfigurations of the surface. The locations of the 16 detectors are shown in Fig. 1b. Multiport matching is taken into consideration to enable optimal power absorption into the distributed detector array ${ }^{33}$. The output signals from the 16 detectors are processed on-chip with a chain of integrated analog amplifiers and filters with variable gain. The collective outputs of the detectors that represent the total power absorbed by the sensing surface can be read in a time-multiplexed fashion. The chip is realized in a $65-\mathrm{nm}$ industry standard digital CMOS process and measures $2 \mathrm{~mm} \times 1 \mathrm{~mm}$ in dimension. The entire chip dissipates less than $10 \mathrm{~mW}$ of DC power.

Mapping of reconfigurable properties to digital states. A logperiodic tooth antenna is used as the sensing surface (see Fig. 1). By moving from a single-port-single-detector system to distributed system, we address three of these reconfigurable properties simultaneously. First, the multiport approach allows us to overcome the bandwidth limitations of the classical 2-port, i.e., single-detector and single-antenna interface. By exploiting mutual interactions of the detectors through the scattering surface, optimal impedances can be synthesized over an order of magnitude higher spectral range. This allows us to increase the BodeFano bandwidth limit by a factor of $N$, where $N$ is the number of detection sites $^{56,57}$ compared with the classical 2-port case. Intuitively, the antenna surface that accepts the incident field and distributes it across the detector sites simultaneously performs the function of the impedance matching across the distributed detector array. In addition to spectral reconfigurability, since the port reconfiguration and power absorption happens at the same place, it allows the surface current to be programmed to shape the reception beam and polarization. The design goal is to translate these digital states to optimal reception against these incident field properties.

Figures 2 and 3 illustrate this. We will discuss the methods of optimization of the detector settings later in the paper, but the figure illustrates the effect of subwavelength reconfiguration to $\mathrm{THz}$ reception properties. In this setup, the chip is abutted by a silicon lens to suppress the substrate modes. However, to avoid beam narrowing and reducing the reconfiguration range of the patterns in a hyper-hemispherical lens, we use a hemispherical lens with only $0.5 \mathrm{~mm}$ of distance kept between the chip and the lens. Figure 2a, b shows the amplitude and phase distribution of the impressed current surface for a linearly polarized incidence at $0.3 \mathrm{THz}$ when the detector settings are optimized for reception for broadside and $45^{\circ}$ incidence, respectively. The simulated reception beams in Fig. $2 \mathrm{c}$ for these settings (and $-45^{\circ}$ incidence) demonstrates the ability to electronically shape the reception 
a

Current $(\mathrm{nA} / \mu \mathrm{m})$

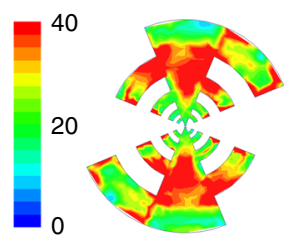

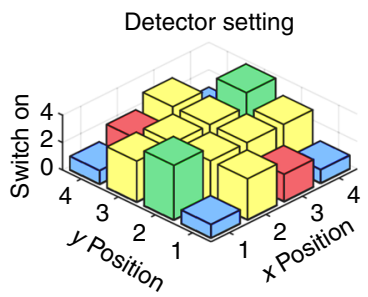

Broadside incidence @ 0.30 THz

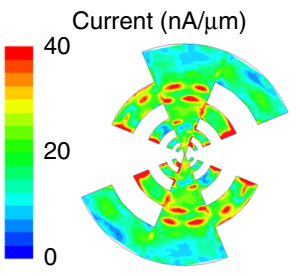

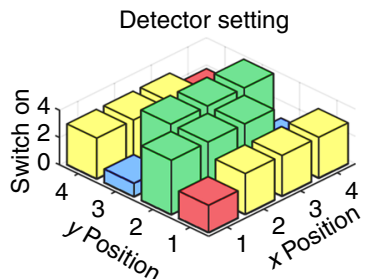

Broadside incidence@ 0.75 THz

b

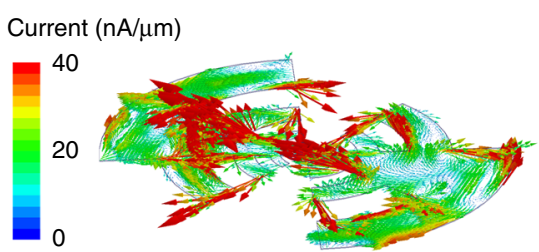

Broadside $x$-Pol @ 0.3 THz

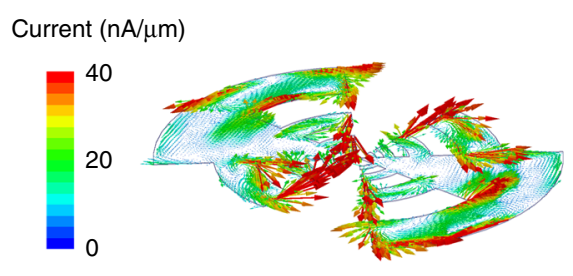

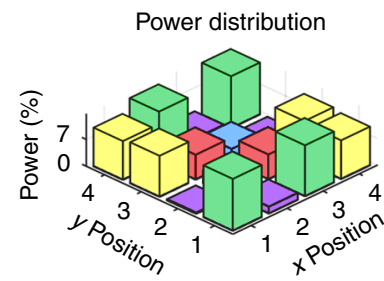

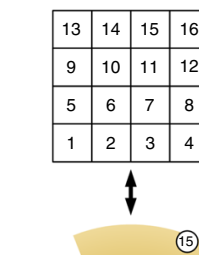
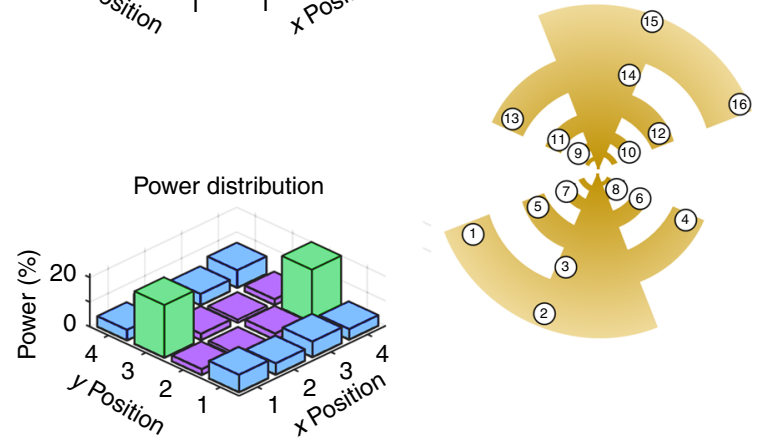

Broadside $y$-Pol @ 0.3 THz
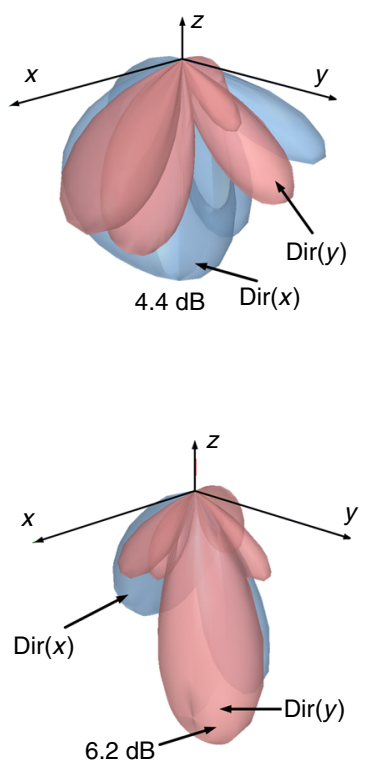

Fig. 3 Direct digital programming of the interface against spectrum and polarization. a Detector configurations, power distributions and surface current configurations for optimized reception at 0.3 and $0.75 \mathrm{THz}$ at the broadside. The figure also shows the mapping of ( $x$, $y$ ) position to location of the

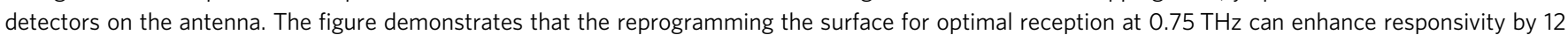
times. b Simulated polarization responsivity and beam patterns when the surface is actuated against the two polarization. Programming the sensor response can enhance the reception by 16 times through polarization rotation of the surface currents

beam and scan it in space. The sensor responsivity indicates the collective rectified response at the output of the detector array distributed over the surface of the electromagnetic structure. This is first electromagnetically simulated with incident field impinging on the distributed antenna loaded with multiport detector array to extract the voltage (local electric field) at each of the detector ports. Enumerating this allows us to calculate the rectified output response of the detector array with nonlinear circuit simulations. Therefore, the simulation takes into account the entire chain from $\mathrm{THz}$ incidence to rectification, including losses in the electromagnetic path, the collective multiport impedance mismatches as well as the detector sensor response at $\mathrm{THz}$ frequencies. This is similar to a phased array operation, but realized with a continuous aperture and multiport subwavelength programming. This technique overcomes the sub- sampling nature of classical phased arrays when operated across a wide-frequency range, where the spacing at the highest end can exceed $\lambda / 2$. This can allow simultaneous reconfigurability against frequency and angles of incidence.

Figure 3 illustrates the optimal reception of broadside incidence at frequencies of 0.30 and $0.75 \mathrm{THz}$ showing the detector settings and frequency dependent power absorption across the detector array. Through reprogramming the surface, the sensor achieves up to 12 times enhancement is reception at $0.75 \mathrm{THz}$ as the incident power gets optimally distributed over the detectors. The state reconfiguration can be extended to even polarization as shown in a 16 times increase in reception for the orthogonal polarization with optimized detector settings.

The mapping of detector settings to the space of incident field properties is non-convex. The power absorbed on the surface in 
a
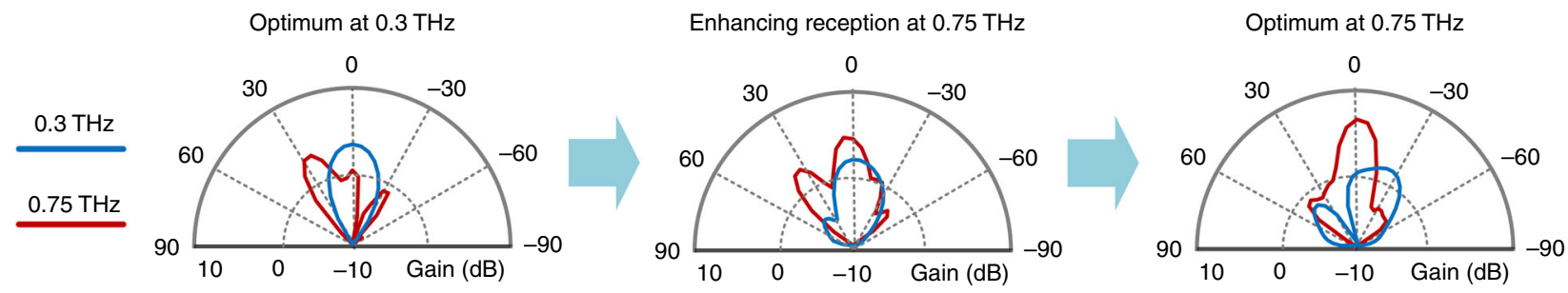

Optimal setting at $0.3 \mathrm{THz}$

Optimal setting at $0.75 \mathrm{THz}$
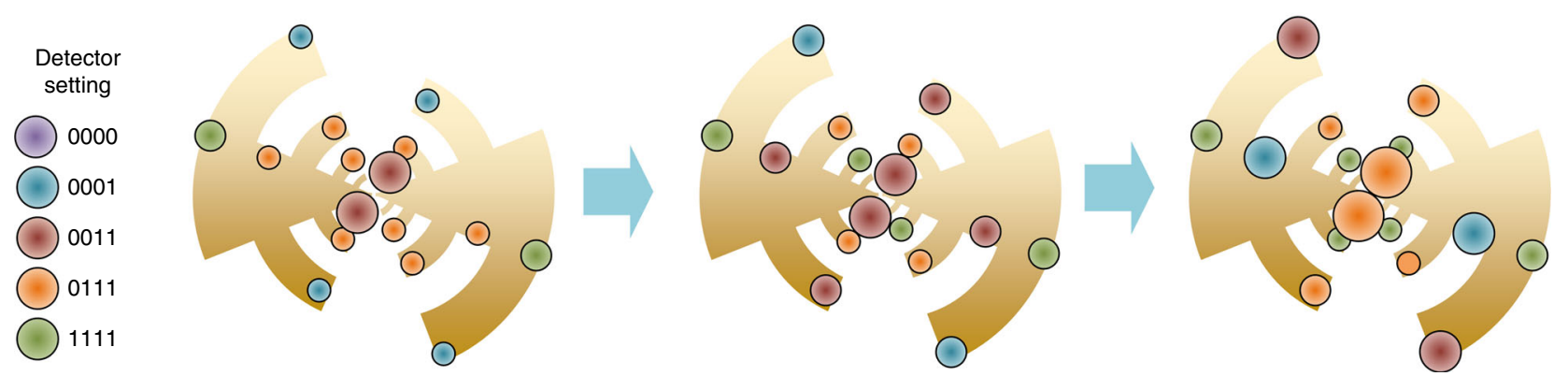

b

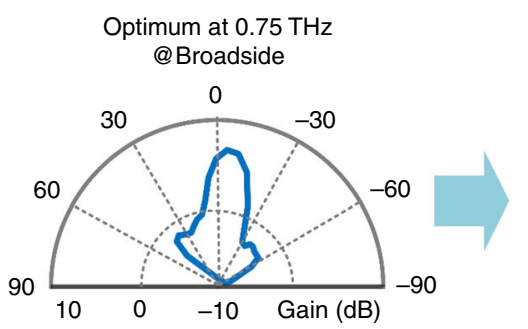

Enhancing reception at $0.75 \mathrm{THz} @ 45^{\circ}$
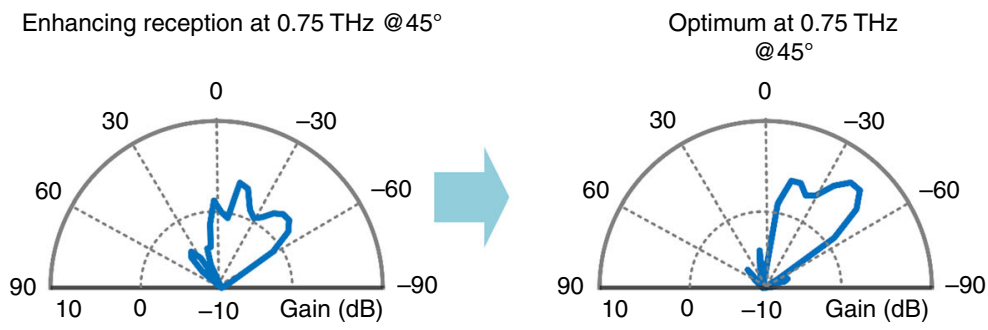

Optimal settings at $0.75 \mathrm{THz}$

Optimum settings at $0.75 \mathrm{THz}$
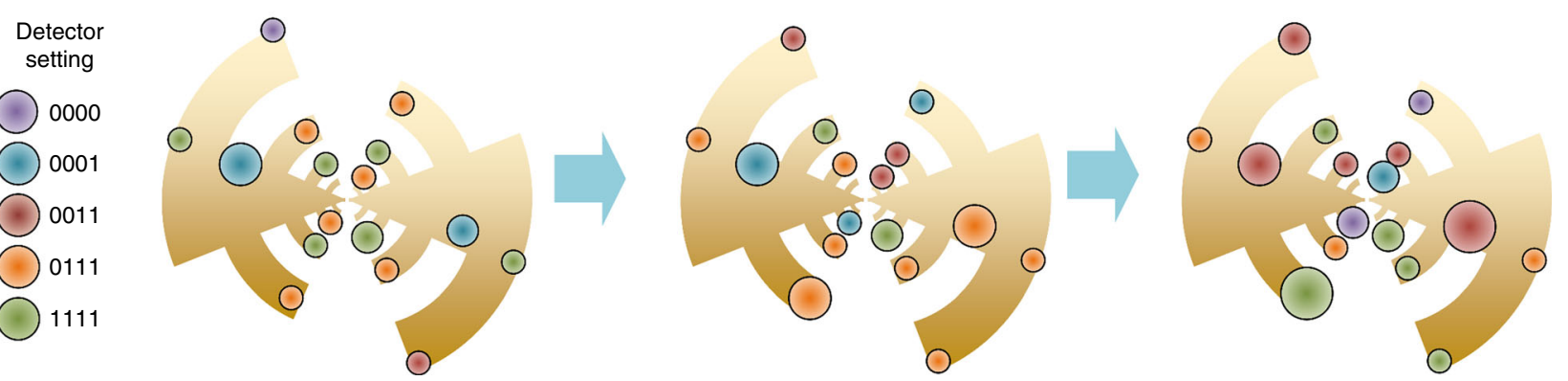

Fig. 4 Mapping the digital electromagnetic states to the incident THz sensor response. a Transformation of the beam patterns as the detector settings are reprogrammed across the 16 -dimensional space from optimal reception from broadside at $0.3-0.75 \mathrm{THz}$. The beam patterns at both the frequencies can be seen to be transformed at the sensor is reprogrammed to shape its $0.75 \mathrm{THz}$ pattern in the broadside. $\mathbf{b}$ Similar transformation of the reception beam at $0.75 \mathrm{THz}$ as the surface is reconfigured from optimal reception at broadside to $45^{\circ}$ incidence at $0.75 \mathrm{THz}$

such a $\mathrm{N}$-port antenna-detector configuration can be derived from the impedance matrices of the passive antenna $\left(\mathbf{Z}_{\text {Ant }} \in\right.$ $\left.\mathbf{R}^{\mathbf{N} \times \mathbf{N}}\right)$, detectors $\left(\mathbf{Z}_{\mathbf{L}} \in \mathbf{R}^{\mathbf{N} \times N}\right)$, and the open circuit voltages $(\mathbf{V} \in$ $\mathbf{R}^{\mathbf{N} \times \mathbf{1})}$ ) excited at the antenna interface under the particular frequency, polarization, and angle of incidence. As a result, the power captured by the antenna, $\left(P_{\text {rec }}\right)$ can be expressed as a function of $\mathbf{Z}_{\mathbf{L}}$ as (Supplementary Notes 1-3)

$$
P_{\text {rec }}=\frac{1}{2} \operatorname{Re}\left(\mathrm{V}^{\mathbf{H}}\left(\mathbf{Z}_{\text {Ant }}^{\mathbf{H}}+\mathbf{Z}_{\mathbf{L}}^{\mathbf{H}}\right)^{-1} \mathbf{Z}_{\mathbf{L}}^{\mathbf{H}}\left(\mathbf{Z}_{\text {Ant }}+\mathbf{Z}_{\mathbf{L}}\right)^{-1} \mathrm{~V}\right)
$$

Therefore, the responsivity of this multiport detector system is simulated combining both electromagnetic and nonlinear circuit simulations. The structure is simulated in a $3 \mathrm{D}$ electromagnetic simulation tool with incident field to calculate its S-parameter and the open circuit voltages. The absorbed power into the individual ports is then calculated by loading the ports with the impedances of the detectors and the capacitor banks. Once the $\mathrm{THz}$ power absorbed at each port is known, the output response of each detector can also be enumerated from the responsivity of each detector through nonlinear circuit simulations (Supplementary Note 1).

Therefore, optimal programming of the $\mathrm{THz}$ surface amounts to searching for a diagonal matrix, $\mathbf{Z}_{\mathbf{L}}$ in a discrete space to maximize $P_{\text {rec }}$. Prior works on electromagnetic optimization in such non-convex and discrete space have utilized genetic algorithm $^{58}$, particle swarm optimization ${ }^{59}$, and alternating direction method of multipliers $(\mathrm{ADMM})^{60}$. Here, we combine gradient decent optimization method with a random search 
a

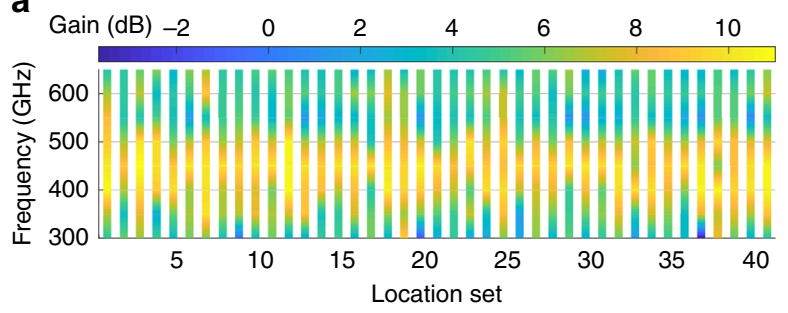

C

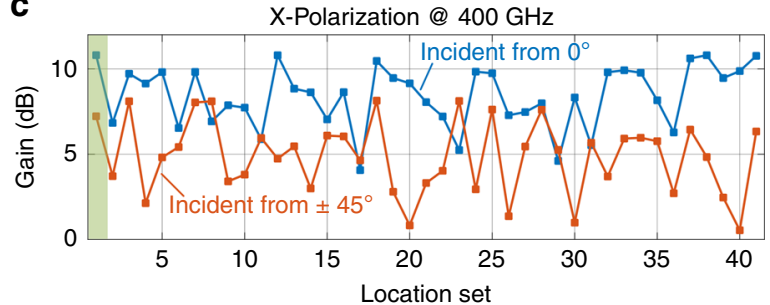

b

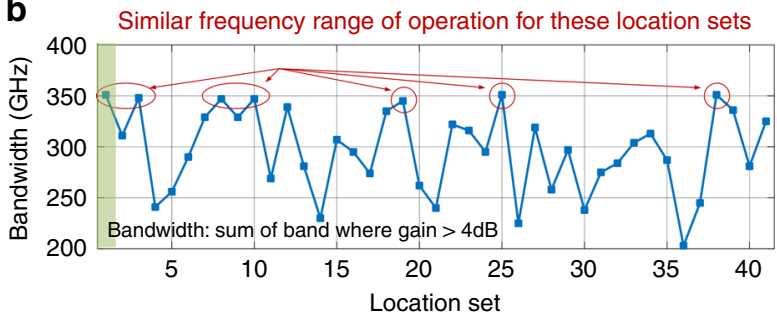

d

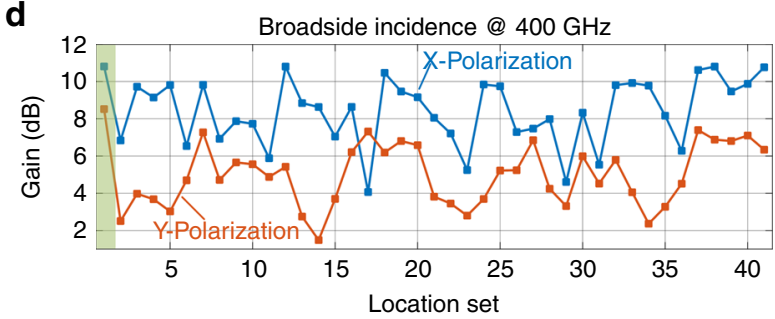

Chosen location set for the detector array position
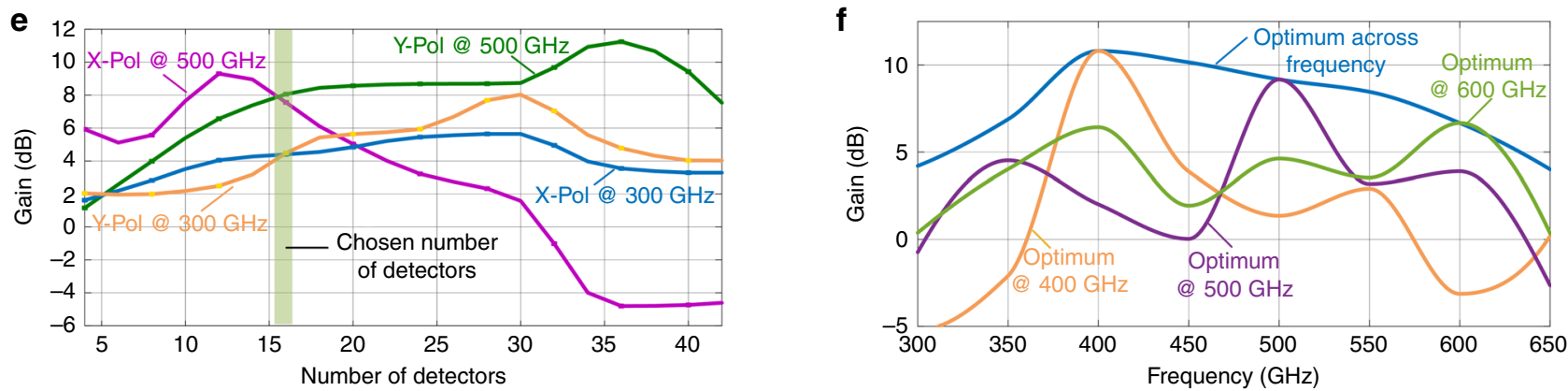

Fig. $5 \mathrm{THz}$ response reconfigurability and its trade-off with the location and number of reconfiguration sites. a Simulated optimized gain with varying locations of the 16 detectors and its effect on peak responsivity and frequency range of operation. $\mathbf{b}$ Simulated variations of bandwidth with location sets of the 16 detectors. The figure demonstrates that more than one solution set can exist to achieve a similar frequency range of operation. $\mathbf{c}$, $\mathbf{d}$ Simulated optimized gain with varying locations of the 16 detectors for different angles of incidence and polarization at $0.4 \mathrm{THz}$. e Simulated gain variations with the number of detectors for various incidence fields. $\mathbf{f}$ For the final design, the figure shows the instantaneous frequency response as the sensor is optimized for various peak frequencies. The optimal reconfigurable sensor response of the chip follows the peak of these individual frequency response curves

algorithm in the 16-dimensional space (formed by the 16 reconfigurable detectors). The discreteness of the space is generated from the digital states of the detectors. Here, optimization is allowed to start with multiple random initial conditions and gradient descent is utilized to obtain the locally optimized solution. In each such iteration, we maximize the total sensor responsivity that is collective response of all the sensors. The process is repeated with a random initial sensor settings to randomize the effect of initial settings.

To further understand the transformation of the $\mathrm{THz}$ sensor properties as the surface is reconfigured, Fig. 4 illustrates the gradual change of the $\mathrm{THz}$ reception pattern from an initial setting to the final optimal one. As shown in Fig. 4a, when the detector setting is optimized for receiving linearly polarized incidence at $0.3 \mathrm{THz}$, the reception beam for $0.3 \mathrm{THz}$ is expectedly broadside. For the same setting, the sensor responsivity toward a broadside incidence at $0.75 \mathrm{THz}$ is, however, poor as shown in the corresponding pattern. The switch settings are color coded and the sizes of the markers qualitatively represent the magnitude of local absorption of the incident power. To demonstrate the transformation of the reception properties as the detectors are optimized for reception at $0.75 \mathrm{THz}$ (from the earlier setting corresponding to $0.3 \mathrm{THz}$ ), we pick an intermediate setting and the corresponding pattern and detector settings are shown. As the surface is programmed, the power distribution and the beam shape at both the frequencies change. The shaping of the beam continues through out the process till it assumes a broadside pattern of $0.75 \mathrm{THz}$. In this case, the reception of the $0.3 \mathrm{THz}$ beam gradually becomes poor, though that does not necessarily have to occur in a single-objective optimization. However, such surface reconfiguration can also be done to assume a frequency selective character that aims to enhance reception at one frequency and minimize that at another frequency. Such a tunable frequency reception across $0.1-1 \mathrm{THz}$ can allow image extraction at discrete frequency slices with high signal-to-noise ratio (SNR). In comparison, in pulse-based broadband imaging, the incident signal is spread over the spectrum and can lead to lower SNR. The shaping of the reception patterns to tilt the beam to a different angle is shown in Fig. 4b. In a manner similar to the previous case, the local field manipulation reprograms the surface current and the spatial distribution of the incident power, and tilts the reception pattern to allow sensitivity alignment to a different angle, similar to a phased array operation.

Design methodology. As shown through the simulated results, the distributed approach allows simultaneous control of the 


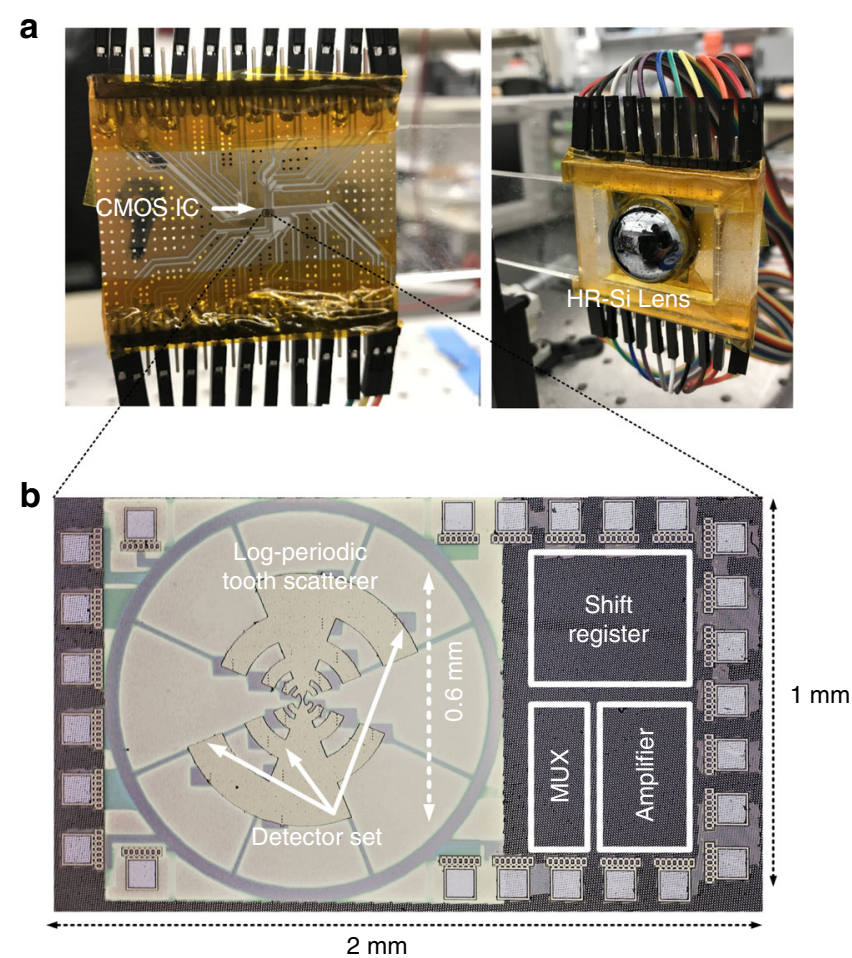

Fig. 6 The chip photo and the packaged system. a The chip is packaged on a printed circuit board supported by a silicon wafer at the back. A highresistivity silicon lens abuts the back of the silicon wafer to enable reception of the $\mathrm{THz}$ waves from the backside. $\mathbf{b}$ The chip is implemented in an industry standard $65-\mathrm{nm}$ CMOS process and measures $2 \mathrm{~mm} \times 1 \mathrm{~mm}$ in dimension

incident field properties, but it also makes the design space interconnected where combination of intuitive design and algorithmic-based approaches have to be relied upon. This is particularly true since the choice of the number of detectors and locations collectively influences the sensor response. We choose the surface as a log-periodic tooth antenna to allow the spectrally distributed resonance over the surface. Of course, this changes when interfaced with the distributed detector array. We follow a heuristic based design methodology to choose the number and location of the detector array. This is illustrated in Fig. 5.

The effect of location of the detector array on the frequency reconfigurability is shown in Fig. 5a. Here, the gain of the aperture $\left(G_{\text {Ant }}\right)$ is defined similar to a single-port antenna as $P_{\text {rec }}=G_{\text {Ant }} P_{\text {flux }} \frac{\lambda^{2}}{4 \pi}$, where $P_{\text {rec }}$ is the power absorbed by the detectors and $P_{\text {flux }}$ is the incident flux at wavelength $\lambda$. We discretize the surface of the radiator into 84 locations at deep subwavelength spacings. We then analyze the effect of the $\mathrm{THz}$ sensor response across frequency, angle of incidence and polarization as we choose a subset of 16 locations from them. The locations of the detectors is chosen with a centro-symmetric distribution, that allows simple rotation of the reception beam by $180^{\circ}$ across the broadside axis by simply rotating the detector settings. The effect of the location distribution is represented in Fig. $5 \mathrm{a}, \mathrm{b}$ as the effective gain of the aperture against frequency for a sample set of 40 locations. For each location set, the performance against frequency is analyzed through optimization of the detector settings. While the effect of the locations on the frequency response is clearly seen, there are multiple location positions that allow similar range of frequency reconfigurability as expected. When analyzed against angle of incidence $\left(0^{\circ}, \pm 45^{\circ}\right)$ and polarization $(X$ and $Y$ ), the location sets show divergent performances. We choose a location set that allow collectively higher gain (and therefore responsivity) across all the three properties, as show in Fig. 5a-d.

The effect of number of detectors is investigated by increasing the number of locations occupied by the detectors up to 84 , and analyzing their reception properties against the incident field. A sampled variation for two frequencies at 300 and $500 \mathrm{GHz}$ and for two polarizations is shown in Fig. 5e. Evidently, lower number of detectors do not show enough reconfigurability across both frequency and polarization. Increasing the number of detectors will load the surface to a large extent effectively reflecting off the incident wave. We choose the number of detectors that allow overall higher response against all the three incident field properties. Once the detector configurations are optimized for a given incident field, Fig. $5 \mathrm{f}$ shows the sensor response as the frequency is varied. The optimally reconfigured sensor response traces the peak of these individual curves. In this design, there is no explicit matching network between the multiport scatterer and multiport detector array, and bandwidth is compared with a single-port antenna with second and fourth order matching networks and the reconfigurable range for the multiport structure far exceeds that of the single-port design (Supplementary Note 3).

There is no provable guarantee on the global optimality of the solution, like in most such heuristic and optimization-based design methodologies. However, in many such methods, the approach allows us to break off from the traditional intuitivebased design processes to incorporate reasonably high performance across a wide range of reconfiguration, difficult to achieve otherwise.

Measurement. The chip is implemented in a $65-\mathrm{nm}$ CMOS process. All measurements are carried out at room temperature. During measurement, the chip is mounted vertically on a Rogers printed circuit board with a high-resistivity silicon wafer at the backside. A high-resistivity silicon lens abuts the wafer at the backside of the substrate to suppress substrate modes and the signal is irradiated from the backside (Fig. 6a). In a manner similar to the simulations, we use a hemispherical lens with a $0.5 \mathrm{~mm}$ distance between the chip and the lens to avoid the sharp narrowing of the beam in a hyper-hemispherical lens setup. The chip occupies an area of $2 \mathrm{~mm} \times 1 \mathrm{~mm}$ as illustrated in Fig. 6b. More details can be found in Supplementary Note 4.

The performance of the chip across the incidence field properties across $0.1-1.0 \mathrm{THz}$ is shown in Figs. 7 and 8. During measurement, optimization to reconfigure against the incident $\mathrm{THz}$ field properties is carried out in the following fashion. At the beginning, the sensor surface is actuated with a random detector array setting through a 64-bit shift register. As the optimization process is executed, sensor outputs are automatically acquired for each setting after they have stabilized. We apply gradient descent optimization on the acquired data on an external computer and the sensor is reprogrammed. The process is repeated with a random initial sensor settings to scramble the effects of initial settings. The entire optimization process is fully automated. In order to reduce the space of the optimization in measurement, we choose three states per detector (instead of five) that corresponds to a total of more than 43 million possible configurations.

The large reconfiguration space is key toward simultaneous optimization against spectrum, angle of incidence and polarization. The measured optimized responsivity and noise-equivalentpower (NEP) across $0.1-1.0 \mathrm{THz}$ for two incidence angles $\left(0^{\circ}\right.$ and $\left.45^{\circ}\right)$ and two polarization $\left(E_{\theta}\right.$ and $\left.E_{\varphi}\right)$ are demonstrated in Fig. 7. 
a
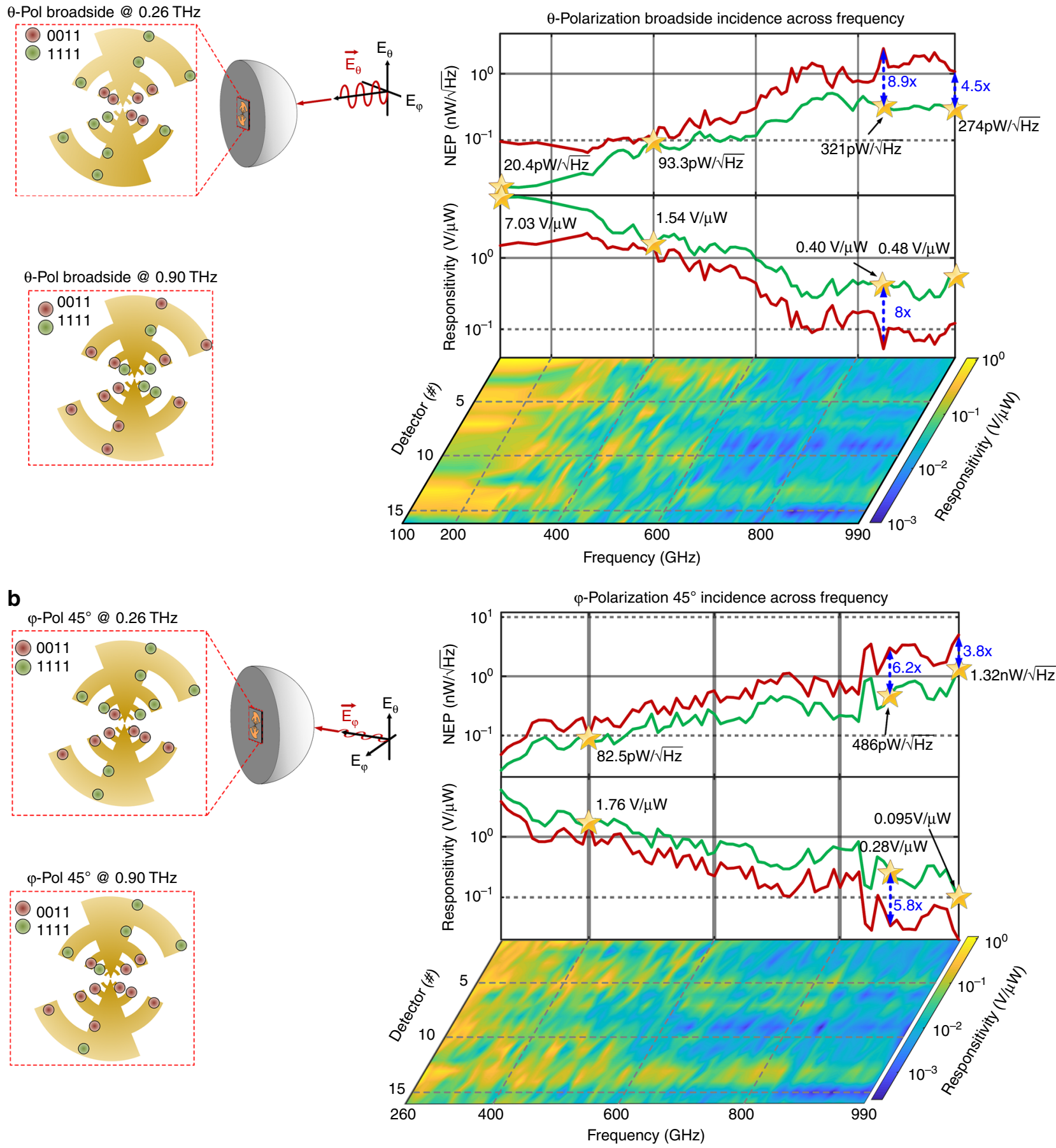

Fig. 7 Measurement results for optimized responsivity and corresponding NEP across $0.1-1.0 \mathrm{THz}$ for two incidence angles at a $0^{\circ}$ and $\mathbf{b} 45^{\circ}$, and two polarizations $\left(E_{\theta}\right.$ and $E_{\varphi}$ ). The figure shows 8.9 and 4.5 times reduction in NEP at $0.86 \mathrm{THz}$ and at $0.99 \mathrm{THz}$ for broadside incidence and 6.2 times reduction in NEP for $45^{\circ}$ incidence at $0.87 \mathrm{THz}$. The green line represents the sensor performance as it is optimized for each incident frequency. The red line represents the performance across frequency for a fixed optimal configuration at $0.4 \mathrm{THz}$. The 2D color plots represent the contribution of power incident absorbed across the detector array across the optimized states between 0.1-1.0 THz. The figure also shows four examples of optimized states for the incident field impinging at two angles of incidence at $0.26 \mathrm{THz}$ and $0.9 \mathrm{THz}$

The figure compares the optimized performance across the frequency range against a static configuration for broadside incidence at $0.4 \mathrm{THz}$. The green line represents the overall sensor performance as the detector states are optimized for each incident frequency. The red line represents the sensor performance across frequency for a fixed optimal configuration at $0.4 \mathrm{THz}$. As can be seen, active reprogramming of the sensing surface allows significant enhancement of sensitivity, including 8.9 and 4.5 times reduction in NEP at 0.86 and $1.0 \mathrm{THz}$ for broadside incidence with $\theta$ polarized waves. We can see a similar enhancement in responsivity and reduction in NEP for $45^{\circ}$ incidence with $\phi$ polarized waves, including a 6.2 times reduction 
a

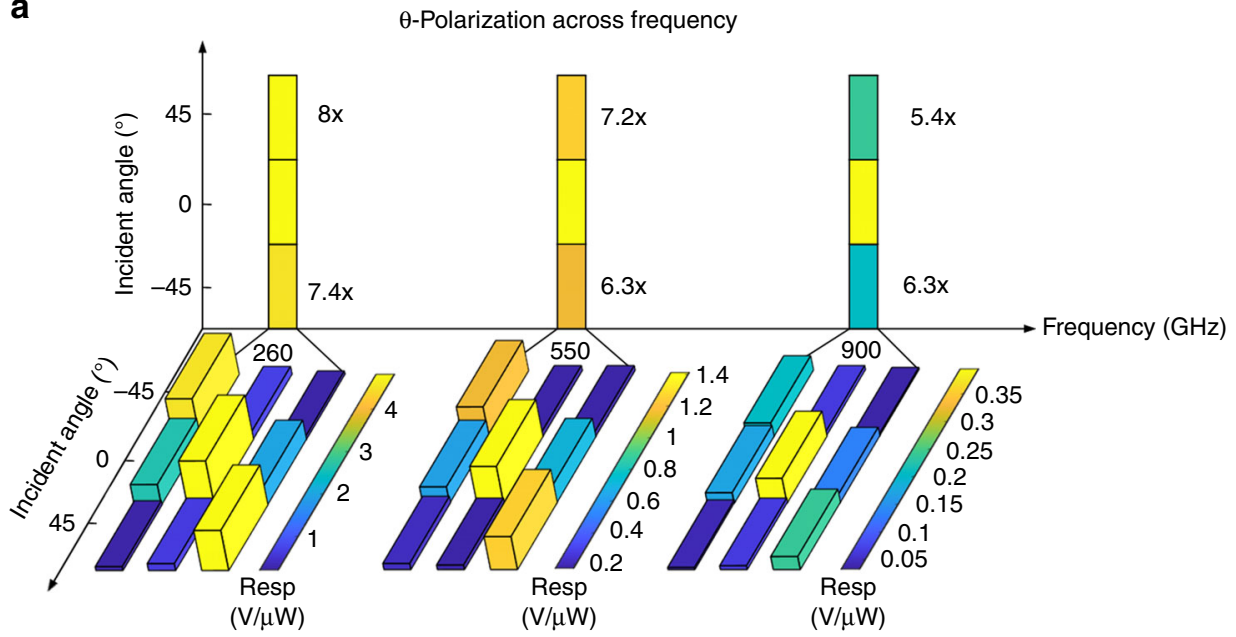

b

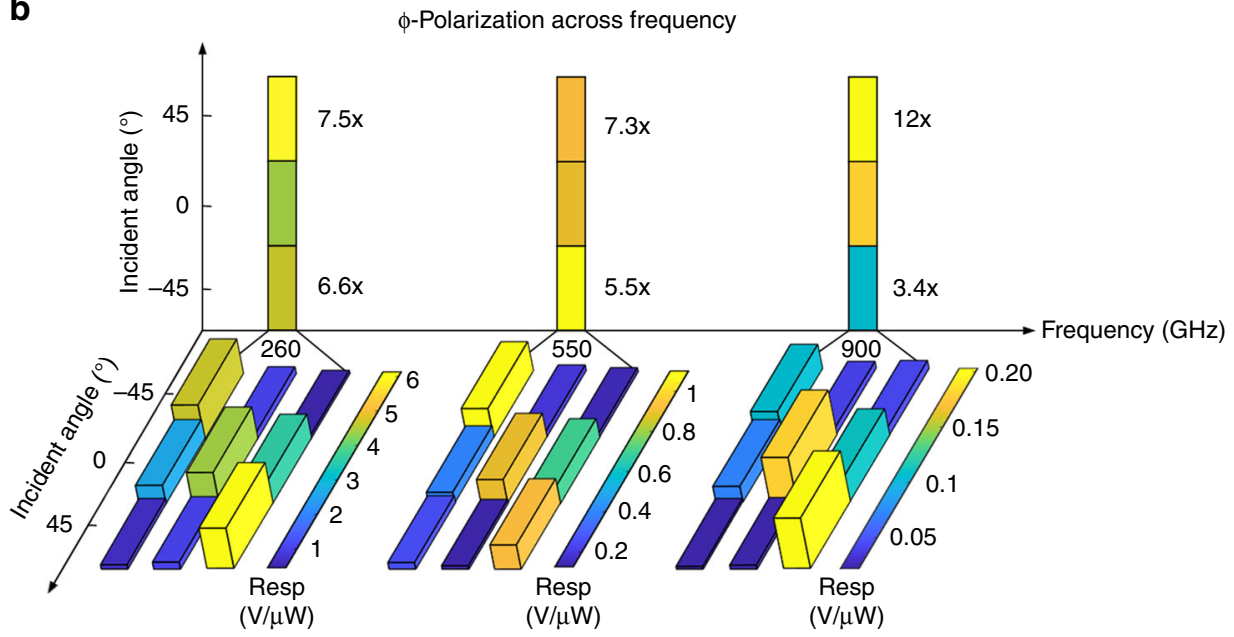

Fig. 8 Measured sensor beam programming with subwavelength reconfiguration. This is measured with varying angles of incidence for two polarizations across $0.1-1.0 \mathrm{THz}$. a Measured optimized performance as the incidence angle is varied for two polarizations across $0.10-1.0 \mathrm{THz}$. For both $E_{\theta}$ and $E_{\varphi}$, the sensitivity can be enhanced significantly by $5-12$ times across $0.10-1.0 \mathrm{THz}$. The horizontal plane shows the instantaneous beam configuration when optimized for reception across the various angles of incidence $\left(45^{\circ}, 0^{\circ}\right.$ and $-45^{\circ}$ ). As can be seen, the reprogramming of the detector states redistributes the surface current to effectively tilt the beam toward the desired angles of incidence. The vertical plane shows the overall responsivitiy enhancement as a result of the reconfiguration when compared against the optimal normal incident state. $\mathbf{b}$ This is also shown in the significant enhancement with reprogramming as the polarization is switched across $E_{\theta}$ to $E_{\varphi}$ across the range

in NEP at $0.87 \mathrm{THz}$. The optimized sensor achieves NEP of $20.4 \mathrm{pW} / \sqrt{\mathrm{Hz}}-274 \mathrm{pW} / \sqrt{\mathrm{Hz}}$ across $0.10-1.0 \mathrm{THz}$ at broadside incidence with $\theta$ polarized waves and $25.9 \mathrm{pW} / \sqrt{\mathrm{Hz}}-$ $1.32 \mathrm{nW} / \sqrt{\mathrm{Hz}}$ at $45^{\circ}$ angle of incidence with $\phi$ polarized waves across $0.26-1.0 \mathrm{THz}$. The figure also shows the spectrally dependent distribution of the absorbed power across the detector array as the incident frequency is varied. The spectrally dependent nature of the distribution of the incident power is evident. The figure also shows the optimized detector settings for the two cases at 0.26 and $0.90 \mathrm{THz}$.

To demonstrate the ability to tilt the beam and reprogram the polarization sensitivity with subwavelength programming, we test the chip by varying the incidence angle for two polarizations, $E_{\theta}$ and $E_{\varphi}$ across $0.10-1.0 \mathrm{THz}$ as shown in Fig. 8. Figure 8a shows the sensor responsivity across angles of incidence as the system is reconfigured successively for incidence angles varying from $-45^{\circ}$ to $45^{\circ}$. Here, we show the responsivity across incidence angles as the beam pattern is reconfigured to tilt from $-45^{\circ}$ to $45^{\circ}$. We also show the overall reconfigured response across angles that allows the sensor to achieve a high response across wide angles of incidence, while being directive for a given configuration. As shown in the Fig. $8 \mathrm{a}$, at $\pm 45^{\circ}$ incidence, the responsivity can be enhanced by 5-12 times nearly reaching similar responsivity as achieved with broadside incidence. The programming of the surface effectively tilts the reception beam towards the angle of incidence allowing electronic scanning across $0.1-1.0 \mathrm{THz}$ for a wide field of view without sacrificing directivity. It is possible to utilize the establishment of such multiple reception beams across the spectrum to create orthogonal measurements for computational-based real-time imaging, though we have not implemented this in this work $^{53}$.

Such reconfiguration can be achieved if we vary the polarization keeping the spectrum and angle of incidence constant. Figure $8 \mathrm{~b}$ shows that the significant enhancement across the frequency range can be observed with reprogramming as we switch the polarization from $E_{\theta}$ to $E_{\varphi}$, reaching up to 12 times as the subwavelength reconfiguration re-orients the beam. The achievable performance obtained across spectrum, angle of 
incidence and polarization, are comparable to a collection of state-of-art sensors, each custom designed within a specified range of these parameters ${ }^{30-32,36,37}$. The experiments demonstrate the ability of such multiport electromagnetic structures with direct programming of the sensing interface to allow for dynamic optimization against incident field properties. Combining these ranges of reconfigurability with sensor fusions can allow for low-cost, robust, versatile, and compact $\mathrm{THz}$ sensing and imaging technology.

\section{Discussion}

The ability to allow a single chip-scale $\mathrm{THz}$ sensor to reconfigure simultaneously against the incident field properties can significantly advance $\mathrm{THz}$ sensing and imaging. The sensing surface, described here, allows reconfiguration for reception across $0.1-1 \mathrm{THz}$ with beam-scanning ability across $\pm 45^{\circ}$ for both polarizations. Being able to sense $\mathrm{THz}$ waves with high sensitivity across 10:1 frequency ratio can enable hyperspectral imaging with both high lateral and depth resolution. Sensor fusions across multiple orthogonal domains (including frequency, beams, and polarization) can allow for superior image registration, and is steadily growing in importance in other spectral domains. This degree of reconfigurability is critical for practical $\mathrm{THz}$ sensing systems, where a single modality interface typically does not suffice. By moving from a single-antenna and single-detector interface to a multiport continuous aperture, it is possible to overcome the grating lobe issues allowing frequency and pattern diversity at the same time. This can allow fast image acquisition, hyperspectral and multi-angle imaging, and computational techniques in the $\mathrm{THz}$ regime for real-time imaging.

The challenge to engineer such programmability is exacerbated by the fact that chip-scale devices are limited in their cutoff frequencies $\left(f_{\max }\right)$. In the implemented chip, the optimal device $f_{\max }$ is $\sim 0.25 \mathrm{THz}$, implying that the devices operate close to four times their cutoff frequencies, when operating near $1 \mathrm{THz}$. Therefore, the presented method that allows such reconfigurable properties at frequencies beyond $f_{\max }$ can be applied to III-V compound semiconductor-base devices to extend operation into higher $\mathrm{THz}$ frequencies $(\sim 3-5 \mathrm{THz})$.

The approach of mapping electromagnetic and sensor properties from a large reconfiguration set also opens up a new design space. By merging multiple functionalities within a single electromagnetic structure (such as, impedance matching and radiating surface in this work), new sensor architectures can emerge. Optimization methods in passive device design, albeit in the photonic domain, has been shown to have yield competitive performance against classical design approaches, but has the potential to open up a new class of optical devices ${ }^{60}$. The computational and distributed subwavelength sensing approach also opens up previously unexplored questions on optimal antenna shapes and detector co-design methodologies in such massively multiport structures, and also new techniques and systems in the optical domain ${ }^{61-63}$. Unlike a static antenna whose shape and boundary condition determine its electromagnetic properties, here, both the structure and the detectors, including their sizing and locations determine the overall responsivity. While we choose a log-periodic structure as the $\mathrm{THz}$ sensing surface, its use for spectral reconfigurability is quite unlike its more traditional use as a wideband interface with a single center port excitation. In fact, in this work, no signal is drawn from the center port at all (see Fig. 1). It can be noted that while in this work we investigate the position, location, and configuration of the detector set for the desired set of reconfigurable parameters based on the choice of the log-periodic tooth surface, an interesting direction for future exploration is the space of co-design of the geometry of the surface and sensory interface.

Given the importance of reconfigurability and sensor fusion for robust $\mathrm{THz}$ imaging and sensing, we expect the space of programmable designs can lead to future $\mathrm{THz}$ sensors with added capabilities. While evidently, this is not as frequency selective as a coherent system, it does overcome the frequency range limited by the tunable nature of such sources in addition to the reconfigurability against incidence angle and polarization. However, this trade-off space is worth investigating for future work to explore further the design parameters and their cross-dependence beyond the ones we have addressed in this work.

The implementation of the chip in a commercial CMOS foundry is a significant advancement in demonstrating such programmable sensors operation up to $1 \mathrm{THz}$. Their operation at room temperature with extremely low power is also important for future applications where power, compactness, and programmability are critical considerations. Eliminating complex optical instrumentation and integration of such programmable functions in a chip-scale form is expected to have significant impact in both technology and application development in the $\mathrm{THz}$ spectrum.

\section{Methods}

Integrated chip implementation and measurement details. Details of the integrated circuit design and the component designs are provided in Supplementary Notes 1, 2 and 3. The log-periodic tooth antenna is realized on a 1.4- $\mu \mathrm{m}$ thick aluminum layer inside the CMOS chip utilizing the embedded metal interconnect layers. The detectors are realized using 65-nm CMOS transistors and the details are shown in Fig. 1 and Supplementary Fig. 1. The biasing voltage and resistors are optimized for minimizing NEP across 0.1-1 THz (Supplementary Figs. 2 and 3). The digitally controllable impedance are realized with capacitor banks with multiple digitally controlled NFETs in parallel as shown in Supplementary Fig. 4. The control signal of the capacitor bank is a 4-bit thermometer code provided by the 64-bit shift register. The chopped rectified $\mathrm{THz}$ signals are processed on-chip with integrated amplifiers (Supplementary Fig. 5). They are realized with integrated transistors as shown in Supplementary Fig. 6. The gain is electronically controllable with a variation of $35 \mathrm{~dB}$ (Supplementary Fig. 7). The multiport antenna structure, its interface with detectors and calculations of power absorption under $\mathrm{THz}$ field incidence is explained in the Supplementary (Supplementary Figs 8 and 9). The measurement setup is shown in Supplementary Fig. 10. The signals are generated with frequency multiplier bank across $0.1-1 \mathrm{THz}$ and across various angles of incidence with a motor-controlled stage. The signal generator is locked to an external $10 \mathrm{MHz}$ signal that provides the reference for lock-in amplifier. The chip is programmed with a Nexs 4 FPGA and the lock-in data is read in a computer through the GPIB ports. The entre system is automated for fully autonomous optimization against the incidence field properties.

\section{Data availability}

The data that support the findings of this study are available from the corresponding author upon reasonable request.

Received: 4 August 2018 Accepted: 27 March 2019

Published online: 20 June 2019

\section{References}

1. Sengupta, K., Nagatsuma, T. \& Mittleman, D. Terahertz integrated electronic and hybrid electronic-photonic systems. Nat. Electron. 1, 622-635 (2018).

2. Duling, I. \& Zimdars, D. Terahertz imaging: revealing hidden defects. Nat. Photonics 3, 630-632 (2009).

3. Song, H. J. \& Nagatsuma, T. Present and future of terahertz communications. IEEE Trans. THz Sci. Technol. 1, 256-263 (2011).

4. Nagatsuma, T., Ducournau, G. \& Renuad, C. Advances in terahertz communications accelerated by photonics. Nat. Photonics 10, 371-379 (2016).

5. Woolard, D. L. et al. Terahertz frequency sensing and imaging: a time of reckoning future applications. Proc. IEEE 93, 1722-1743 (2005).

6. Pickwell, E. \& Wallace, V. P. Biomedical applications of terahertz technology. J. Phys. D. Appl. Phys. 39, R301-R310 (2006). 
7. Liu, H.-B. et al. Terahertz spectroscopy and imaging for defense and security applications. Proc. IEEE 95, 1514-1527 (2007).

8. Xie, L. et al. Extraordinary sensitivity enhancement by metasurfaces in terahertz detection of antibiotics. Nat. Sci. Rep. 77, 4049-4051 (2015).

9. Cooper, K. B. et al. THz imaging radar for standooff personnel screening. IEEE Trans. THz Sci. Technol. 1, 169-182 (2011).

10. Stake, J., Malko, A., Bryllert, T. \& Vukusic, J. Status and prospects of highpower heterostructure barrier varactor frequency multipliers. Proc. IEEE 105, 1008-1019 (2017).

11. Urteaga, M., Griffith, Z., Seo, M., Hacker, J. \& Rodwell, M. J. W. InP HBT technologies for THz integrated circuits. Proc. IEEE 105, 1051-1067 (2017)

12. Chevalier, P. et al. $\mathrm{Si} / \mathrm{SiGe}: \mathrm{C}$ and $\mathrm{InP} / \mathrm{GaAsSb}$ heterojunction bipolar transistors for THz applications. Proc. IEEE 105, 1035-1050 (2017).

13. Voinigescu, S. P. et al. Silicon millimeter-wave, terahertz, and high-speed fiber-optic device and benchmark circuit scaling through the 2030 ITRS horizon. Proc. IEEE 105, 1087-1104 (2017).

14. Schröter, M. et al. SiGe HBT technology: future trends and TCAD-based roadmap. Proc. IEEE 105, 1068-1086 (2017).

15. Ju, L. et al. Graphene plasmonics for tunable terahertz metamaterials. Nat. NanoTech. 6, 630-634 (2011).

16. Tassin, P. et al. Graphene for terahertz applications. Science 341, 620-621 (2013).

17. Vijayraghavan, K. et al. Broadly tunable terahertz generation in mid-infrared quantum cascade lasers. Nat. Comm. 4, 2021 (2013).

18. Sirtori, C., Barieri, S. \& Colombelli, R. Wave engineering with THz quantum cascade lasers. Nat. Photon. 7, 691-701 (2013).

19. Wanke, M. C. et al. Monolithically integrated solid-state terahertz transceivers. Nat. Photon. 4, 565-569 (2010).

20. Hammar, A. et al. Terahertz direct detection in $\mathrm{YBa}_{2} \mathrm{Cu}_{3} \mathrm{O}_{7}$ microbolometers. IEEE Trans. THz Sci. Technol. 1, 390-394 (2011).

21. Liu, L. et al. Development of integrated terahertz broadband detectors utilizing superconducting hot-electron bolometers. IEEE Trans. Appl. Supercond. 19, 282-286 (2009).

22. Peng, K. et al. Single nanowire photoconductive terahertz detectors. Nano. Lett. 15, 206-210 (2014).

23. Grady, N. K. et al. Terahertz Metamaterials for Linear Polarization Conversion and Anomalous Refraction Science 340, 1304-1307 (2013).

24. Tanoto, H. et al. Greatly enhanced continuous-wave terahertz emission by nano-electrodes in a photoconductive photomixer. Nat. Photon. 6, 121-126 (2012).

25. Heshmat, B. et al. Nanoplasmonic terahertz photoconductive switch on GaAs. Nano. Lett. 12, 6255-6259 (2012).

26. Huang, D. et al. $324 \mathrm{GHz}$ CMOS frequency generator using linear superposition technique. IEEE Int. Solid-State Circuits Conf. Dig. Tech. Papers (ISSCC), 476-477 (2008).

27. Hu, Z., Kaynak, M. \& Han, R. High-power radiation at $1 \mathrm{THz}$ in silicon: a fully scalable array using a multi-functional radiating mesh structure. IEEE J. SolidState Circuits 53, 1313-1327 (2018).

28. Sengupta, K. \& Hajimiri, A. $0.28 \mathrm{THz}$ power-generation and beam-steering array in CMOS based on distributed active radiators. IEEE J. Solid-State Circuits 47, 3013-3031 (2012).

29. Momeni, O. \& Afshari, E. High power terahertz and millimeter-wave oscillator design: a systematic approach. IEEE J. Solid-State Circuits $\mathbf{4 6}$, 583-597 (2011).

30. Liu, Z. Y., Liu, L. Y., Yang, J. \& Wu, N. J. A CMOS fully integrated $860-\mathrm{GHz}$ terahertz sensor. IEEE Trans. THz Sc. Techn. 7, 455-465 (2017).

31. Han, R. et al. Active terahertz imaging using Schottky diodes in CMOS: array and 860-GHz pixel. IEEE J. Solid-State Circuits 48, 2296-2308 (2013).

32. Hadi, R. et al. A 1 k-pixel video camera for 0.7-1.1 terahertz imaging applications in 65-nm CMOS. IEEE J. Solid-State Circuits 47, 2999-3012 (2012).

33. Wu., X. \& Sengupta, K. On-chip THz spectroscope exploiting electromagnetic scattering with multi-port antenna. IEEE J. Solid-State Circuits 51, 3049-3062 (2016).

34. Wu, X. \& Sengupta, K. Single-chip source-free terahertz spectroscope across 0.04-0.99 THz: combining sub-wavelength near-field sensing and regression analysis. Opt. Exp. 26, 7163-7175 (2018).

35. Wu, X. \& Sengupta, K. Wide-band $\mathrm{THz}$ spectroscope in silicon $\mathrm{THz}$ combining sub-wavelength near-field sensing and robust regression analysis. Intl. Microw. Symp. 1468-1471 (2018).

36. Schuster, F. et al. Broadband terahertz imaging with highly sensitive silicon CMOS detectors. Opt. Express 19, 7827-7832 (2011).

37. Sengupta, K., Seo, D. J., Yang, L. \& Hajimiri, A. Silicon integrated $280 \mathrm{GHz}$ imaging chipset with $4 \times 4$ SiGe receiver array and CMOS source. IEEE Trans. THz Sc. Techn. 5, 427-437 (2015).

38. Dyakonov, M. \& Shur, M. Shallow water analogy for a ballistic field effect transistor: New mechanism of plasma wave generation by DC current. Phys. Rev. Lett. 71, 2465-2468 (1993).
39. Xu, Z. \& Lam, E. Y. Image reconstruction using spectroscopic and hyperspectral information for compressive terahertz imaging. J. Opt. Soc. Am. A. 27, 1638-1646 (2010).

40. Hong, L. \& Sengupta, K. Fully Integrated optical spectrometer between 500 $\mathrm{nm}-830 \mathrm{~nm}$ in CMOS. IEEE Trans. Biomed. Circuits Syst. (Spec. ISSCC issue) 11, 1176-1191 (2017).

41. Hong, L. \& Sengupta, K. Fully integrated optical spectrometer with 500-to$830 \mathrm{~nm}$ range in $65 \mathrm{~nm}$ CMOS. IEEE Intl. Conf. Solid-State Circuits Conf. 462-463 (2017).

42. Sensale-Rodriguez, B., Yan, R., Liu, L., Jena, D. \& Xing, H. G. Graphene for reconfigurable terahertz optoelectronics. Proc. IEEE 101, 1705-1716 (2013).

43. Tao, H. et al. Reconfigurable terahertz metamaterials. Phys. Rev. Lett., 103, 147401 (2009).

44. Chen, H.-T. et al. Experimental demonstration of frequency-agile terahertz metamaterials. Nat. Photon. 2, 295-298 (2008).

45. Berry, C. W., Moore, J. \& Jarrahi, M. Design of reconfigurable metallic slits for terahertz beam modulation. Opt. Exp. 19, 1236-1245 (2011).

46. Liu, C., Ye, J. \& Zhang, Y. Thermally tunable THz filter made of semiconductors. Opt. Comm. 283, 865-868 (2010).

47. Sanphuang, V., Ghalichechian, N., Nahar, N. K. \& Volakis, J. L. Reconfigurable $\mathrm{THz}$ filters using phase-change material and integrated heater. IEEE Trans. THz. Sci. Technol. 6, 583-591 (2016).

48. Huang, Y., Wu, L.-S., Tang, M. \& Mao, J. Design of a beam reconfigurable $\mathrm{THz}$ antenna with graphene-based switchable high-impedance surface. IEEE Trans. Nano. 11, 836-842 (2012).

49. Wang, X.-C., Zhao, W.-S., Hu, J. \& Yin, W.-Y. Reconfigurable terahertz leakywave antenna using graphene-based high-impedance surface. IEEE Trans. Nano. 14, 62-69 (2015).

50. Xu, Z., Dong, X. \& Bornemann, J. Design of a reconfigurable MIMO system for $\mathrm{THz}$ communications based on graphene antennas. IEEE Trans. THz Sci. Technol. 4, 609-617 (2014).

51. Wu, X. \& Sengupta, K. A programmable active THz electromagnetic surface on-chip for multi-functional imaging. Intl. Microw. Symp. 1464-1467 (2018).

52. Watts, C. M. et al. Terahertz compressive imaging with metamaterial spatial light modulators. Nat. Photon. 8, 605-609 (2014).

53. Hunt, J. et al. Metamaterial apertures for computational imaging. Sciece 339, 310-313 (2013).

54. Sadhu, B. et al. A 28-GHz 32-element TRX phased-array IC with concurrent dual-polarized operation and orthogonal phase and gain control for $5 \mathrm{G}$ communications. IEEE J. Solid-State Circuits 52, 3373-3391 (2017).

55. Sun, J. et al. Large-scale nanophotonic phased array. Nature 493, 195-199 (2013).

56. Nie, D. \& Hochwald, B. M. Improved broadband matching bound. IEEE Trans. Antenna Propag. 65, 5878-5885 (2017).

57. Nie, D. \& Hochwald, B. M. Broadband matching bounds for coupled loads. IEEE Trans. Circuits Syst. 62, 995-1004 (2015).

58. Rahmat-Samii, Y. \& Michielssen, E. Electromagnetic Optimization by Genetic Algorithms. (Wiley \& Sons, NY, 1999).

59. Robinson, J. \& Rahmat-Samii, Y. Particle swarm optimization in electromagnetics. IEEE Trans. Antenna Propag. 52, 397-407 (2004).

60. Piggott, A. Y. et al. Inverse design and demonstration of a compact and broadband on-chip wavelength demultiplexer. Nat. Photon. 9, 374-378 (2015).

61. Hong, L., Li, H., Yang, H. \& Sengupta, K. Fully integrated fluorescence biosensors on-chip employing multi-functional nanoplasmonic optical structures in CMOS. IEEE J. Solid-State Circuits 52, 2388-2406 (2017)

62. Hong, L., Li, H., Yang, H. \& Sengupta, K. Nano-plasmonics and electronics co-integration in CMOS enabling a pill-sized multiplexed fluorescence microarray system. Biomed. Opt. Exp. 9, 5735-5758 (2018).

63. Hong, L., Li, H., Yang, H. \& Sengupta, K. Integrated copper-based subwavelength angle insensitive nano-plasmonic filters for ultra-miniaturized fluorescence microarray in a $65-\mathrm{nm}$ digital CMOS process. Acs. Photonics 5, $4312-4322$ (2018)

\section{Acknowledgements}

The authors would like to acknowledge National Science Foundation (ECCS-1408490, ECCS-1509560), Young Investigator Program (YIP) (N000141712494), DURIP program (N000141612655) and grant (N000141512217) from Office of Naval Research, and the Multi University Research Initiative (MURI) program (FA9550-16-1-0566) from Air Force Office of Scientific Research for funding.

\section{Author contributions}

X.W. and K.S. designed the experiments. X.W. and H.L. measured the chips. X.W. and K.S. contributed to writing the manuscript. 


\section{Additional information}

Supplementary Information accompanies this paper at https://doi.org/10.1038/s41467019-09868-6.

Competing interests: The authors declare no competing interests.

Reprints and permission information is available online at http://npg.nature.com/ reprintsandpermissions/

Journol peer review information: Nature Communications thanks Patrick Reynaert and other anony-mous reviewers for their contribution to the peer review of this work.

Publisher's note: Springer Nature remains neutral with regard to jurisdictional claims in published maps and institutional affiliations. (c) (i) Open Access This article is licensed under a Creative Commons Attribution 4.0 International License, which permits use, sharing, adaptation, distribution and reproduction in any medium or format, as long as you give appropriate credit to the original author(s) and the source, provide a link to the Creative Commons license, and indicate if changes were made. The images or other third party material in this article are included in the article's Creative Commons license, unless indicated otherwise in a credit line to the material. If material is not included in the article's Creative Commons license and your intended use is not permitted by statutory regulation or exceeds the permitted use, you will need to obtain permission directly from the copyright holder. To view a copy of this license, visit http://creativecommons.org/ licenses/by/4.0/.

(C) The Author(s) 2019 